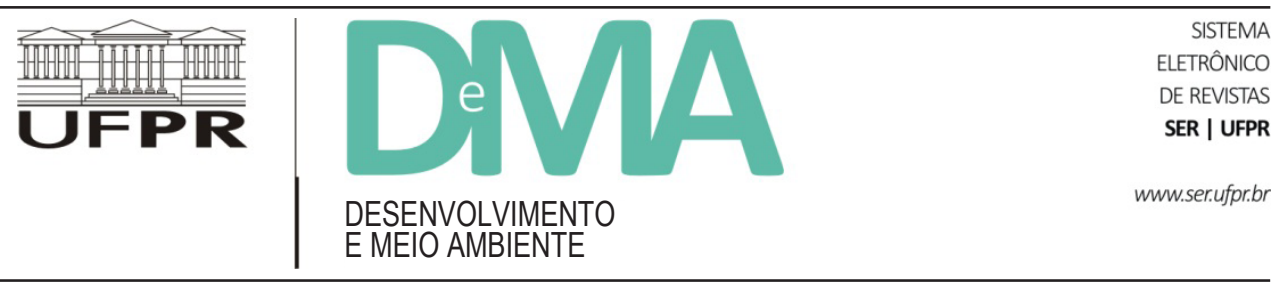

\title{
Fortalezas e fragilidades do Sistema de Unidades de Conservação Potiguar
}

\section{Strengths and Weaknesses of the Potiguar Protected Areas}

\author{
Marília Gomes TEIXEIRA ${ }^{1}$, Eduardo Martins VENTICINQUE ${ }^{2}$ \\ ${ }^{1}$ Bióloga (UFRN). Programa de Pós Graduação em Ecologia. Universidade Federal do Rio Grande do Norte (UFRN). Departamento de \\ Ecologia. E-mail: mariliabgt@hotmail.com \\ ${ }^{2}$ Doutor em Ciências Biológicas (UNESP). Professor da Universidade Federal do Rio Grande do Norte (UFRN), Departamento de Ecologia. \\ E-mail: eduardo.venticinque@gmail.com
}

Artigo: Recebido em: 22 de julho de 2013; Versão final aceita em: 1 de abril de 2014.

RESUMO O Rio Grande do Norte é um dos estados que ainda não foi contemplado com o método de Avaliação Rápida e Priorização da Gestão de Unidades de Conservação-Rapid Assessment and Prioritization of Protected Area Management (RAPPAM). Com isso, o presente trabalho visa aplicar o RAPPAM para identificar as fragilidades e fortalezas na implementação do Sistema de Unidades de Conservação Potiguar. No geral, as Unidades de Conservação (UCs) avaliadas obtiveram uma nota mediana de 49\% para efetividade de gestão, não apresentando diferença quanto ao grau de implementação entre as UCs de Proteção Integral e Uso Sustentável, assim como entre as UCs sobre gestões estadual e federal. As fragilidades do Sistema de Unidades de Conservação Potiguar se refletem principalmente na carência de investimento em recursos humanos, financeiros e de pesquisa, na falta de aplicação dos princípios da sustentabilidade na utilização dos recursos e no pouco comprometimento social e político em manter um sistema de áreas protegidas conectadas. Contudo, encontra potencialidades no momento em que conta com um planejamento operacional que abrange tanto valores sociais como econômicos e ambientais, que é otimizado com a implantação de uma boa infraestrutura física da UC, facilidade de comunicação entre os diferentes atores sociais envolvidos na gestão e na participação comunitária representada pelos Conselhos gestores.

Palavras-chave: Rio Grande do Norte; Unidades de Conservação; RAPPAM.

ABSTRACT Rio Grande do Norte is one of the states that have not yet been awarded a rating of Rapid Assessment and Prioritization of Protected Area Management (RAPPAM). Thus, this study aims at applying the RAPPAM to identify weaknesses and strengths in the implementation of the System of Potiguar Conservation Units. Overall evaluated Conservation Units (UCs) obtained an average score of $49 \%$ for Management Effectiveness, showing no difference in the degree of implementation among the UCs Full Protection and Sustainable Use, as well as among the UCs on State and Federal management. Weaknesses of the System of Potiguar Conservation Units reflected mainly in the lack of investment in human, financial and research resources, as well as in the lack of 
application of the sustainability principles in the use of resources, and in little social and political commitment to maintain a system of protected areas connected. However it finds potential when it counts on an operational plan that covers social, economic and environmental values, which is optimized with the implementation of a good physical infrastructure of UC, as well as the ease of communication between the different actors involved in the management and the community participation represented by Council managers.

Keywords: Rio Grande do Norte; Protected Areas; RAPPAM.

\section{Introdução}

A criação de áreas legalmente protegidas sob a forma de Unidades de Conservação (UCs) tem se tornado uma estratégia fundamental para a conservação da biodiversidade. Segundo Schaik e Rijksen (2002), os benefícios advindos da conservação são visíveis a longo prazo e raramente mensuráveis. De acordo com Prates (2012) o Brasil apresenta hoje 1.698 UCs sob gestões federal, estadual, municipal e particular, abrangendo uma área de 1,43 milhão de $\mathrm{km}^{2}$, onde estão representados $16,8 \%$ do território continental.

A ausência da regularização fundiária; de uma base institucional sólida; a presença dos conflitos socioambientais com a população do interior e entorno das UCs, devido ao uso e ocupação da área; a falta de recursos humanos e financeiros são, de acordo com Paduá (2011), as principais ameaças que comprometem a implantação e consequente consolidação das Unidades de Conservação no Brasil.

A avaliação da efetividade de manejo é um instrumento de gestão de áreas protegidas indispensável, uma vez que mede a priori como as UCs estão sendo eficientes em alcançar seus objetivos de conservação. Hoje há diversas ferramentas de avaliação de gestão de áreas protegidas, tais como: Rapid Assessment and Prioritization of Protected Area Management - Avaliação Rápida e Priorização do Manejo de Unidades de Conservação (RAPPAM) (Ervin, 2003); Medición de la Efectividad del Manejo de Areas Protegidas (Cifuentes et al., 2000); Management Effectiveness Tracking Tool (Hocking et al., 2000); e Scorecard (TNC, 2007).

O RAPPAM foi a ferramenta de gestão que apresentou maior popularização e aplicação no mundo. No Brasil, esta metodologia foi utilizada primeiramente para avaliar as UCs de São Paulo (WWF-Brasil \& Instituto Florestal, 2004), posteriormente para avaliar as UCs
Federais do Brasil (WWF-Brasil \& IBAMA, 2007) Acre (WWF-Brasil, SEMA-AC, SEF-AC \& ICMBIO, 2009), Amapá (WWF-Brasil, IEF-AP, SEMA-AP \& ICMBIO, 2009), Mato Grosso (WWF-Brasil, SEMA-MT \& ICMBIO, 2009), Amazonas (WWF-Brasil, SDS-AM \& ICMBIO, 2011), Pará (WWF-Brasil, SEMA-PA \& ICMBIO, 2011), Rondônia (WWF-Brasil, SEDAM-RO \& ICMBIO, 2011), Mato Grosso do Sul (WWF-Brasil \& IMASUL, 2011) e novamente as UCs Federais (WWF-Brasil \& ICMBIO, 2012).

O Rio Grande do Norte é um dos estados que ainda não foi contemplado com a Avaliação do RAPPAM. Em seus 52.810,699 $\mathrm{km}^{2}$ (IBGE, 2010) possui apenas 2,16\% de sua área protegida sob a forma de UCs, das quais oito são de Proteção Integral, treze de Uso Sustentável e uma não categorizada de acordo com a lei do Sistema Nacional de Unidades de Conservação. Destas, cinco são administradas pelo Instituto Chico Mendes de Conservação da Biodiversidade (ICMBio) a nível federal, nove são administradas pelo Instituto de Desenvolvimento Sustentável e Meio Ambiente do Rio Grande do Norte (IDEMA) a nível estadual, cinco são Reservas Particulares de Patrimônio Natural (RPPN's), sendo administradas pelos seus proprietários, e três estão sob gestão municipal de Natal, Ceará-Mirim e Tibau do Sul (Figura 1 e Tabela 1).

É esperado que as áreas protegidas sob a forma de UC no Estado se expandam significativamente nos próximos anos. De acordo com Farias \& Medeiros (2012), as Unidades de Conservação sob gestão estadual que estão em processo de criação são: Área de Proteção Ambiental Dunas do Rosado, Área de Proteção Ambiental das Carnaúbas, Parque Estadual Mangues do Potengi, Monumento Natural Morro do Careca, Parque Estadual do Jiqui e Área de Proteção Ambiental Cavernas de Martins. Todavia, é necessário a priori garantir que as atuais Unidades de Conservação sejam consolidadas e eficientes em alcançar seu objetivo de conservação. 


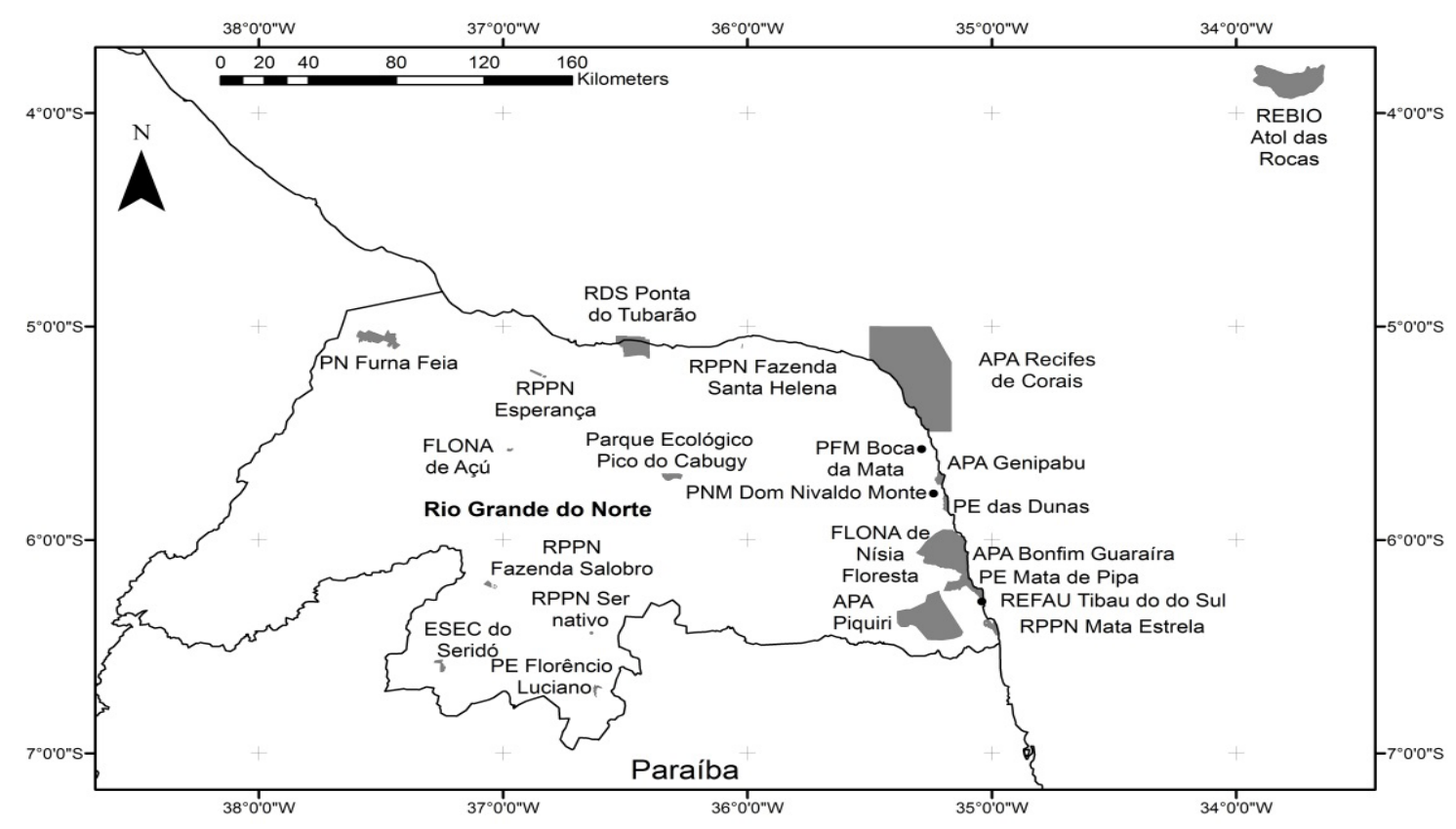

FIGURA 1 - Unidades de Conservação do Rio Grande do Norte. Estação Ecológica (ESEC), Parque Nacional (PN), Reserva Biológica (REBIO), Parque Estadual (PE), Parque Natural Municipal (PNM), Parque Florestal Municipal (PFM), Floresta Nacional (FLONA), Área de Proteção Ambiental (APA), Reserva de Desenvolvimento Sustentável (RDS), Reserva Faunística (REFAU) e Reserva Particular de Patrimônio Natural (RPPN). No caso do Parque Natural Municipal Dom Nivaldo Monte, da Reserva Faunística de Tibau do Sul e do Parque Florestal Municipal Boca da Mata, sua localização é feita apenas por um ponto, por não estar disponível o polígono que define sua área.

O presente trabalho propõe: a) identificar as fragilidades e fortalezas da implementação do Sistema de Unidades de Conservação Potiguar; b) apontar as pressões e ameaças que comprometem o manejo das Unidades de Conservação; c) comparar o grau de implementação entre as Unidades de Conservação de Uso Sustentável e Proteção Integral; d) confrontar o nível de implementação entre as Unidades de Conservação Estaduais e Federais; e e) Indicar as ações prioritárias para melhorar o grau de implementação do Sistema de Unidades de Conservação do RN.

\section{Metodologia}

Para averiguar o grau de implementação das UCs do Rio Grande do Norte, fez-se uso da Avaliação Rápida e Priorização da Gestão de Unidades de Conservação-Rapid Assessment and Prioritization of Protected Area Management (RAPPAM) (Ervin, 2003). O modelo do questionário RAPPAM e a análise dos dados foi o mesmo utilizado pela WWF-Brasil, modificado de Ervin (2003) (Tabela 2). 
TABELA 1 - UNIDADES DE CONSERVAÇÃO DO RIO GRANDE DO NORTE. ESTAÇÃO ECOLÓGICA (ESEC), PARQUE NACIONAL (PN), RESERVA BIOLÓGICA (REBIO), PARQUE ESTADUAL (PE), PARQUE NATURAL MUNICIPAL (PNM), PARQUE FLORESTAL MUNICIPAL (PFM), FLORESTA NACIONAL (FLONA), ÁREA DE PROTEÇÃO AMBIENTAL (APA), RESERVA DE DESENVOLVIMENTO SUSTENTÁVEL (RDS), RESERVAFAUNÍSTICA(REFAU), RESERVA PARTICULAR DE PATRIMÔNIO NATURAL (RPPN), INSTITUTO CHICO MENDES DE CONSERVAÇÃO DA NATUREZA (ICMBIO) E INSTITUTO DE DESENVOLVIMENTO SUSTENTÁVEL E MEIO AMBIENTE DO RN (IDEMA).

\begin{tabular}{|c|c|c|c|}
\hline $\mathbf{U C}$ & Área (ha) & Criação & Responsável \\
\hline ESEC Seridó & 1.124 & Decreto Federal n. ${ }^{\circ} 87.222$ de 31.05 .82 & ICMBio \\
\hline PN da Furna Feia & 8.494 & Decreto Federal $n^{\circ} 4.340$ de 5.07.2012 & ICMBio \\
\hline REBIO Atol das Rocas & 36.249 & Decreto Federal n. ${ }^{\circ} 83.549$ de 05.06 .79 & ICMBio \\
\hline PE Dunas de Natal & 1.172 & Decreto Estadual n. ${ }^{\circ} 7.237$ de 22.11 .77 & IDEMA \\
\hline PE Florêncio Luciano & 446,02 & Decreto Estadual n. ${ }^{\circ} 10.120$ de 10.08 .88 & IDEMA \\
\hline PE Mata de Pipa & 290,88 & $\begin{array}{l}\text { Decreto Estadual } \mathrm{n}^{\circ} 19.341 \mathrm{de} \\
12.09 .2006\end{array}$ & IDEMA \\
\hline PNM Dom Nivaldo Monte & 62,20 & Decreto Municipal no 8.078 de 13.12. 2006 & Prefeitura de Natal \\
\hline PFM Boca da Mata & 68,90 & Decreto Municipal 2.132, de 23.12.2008 & $\begin{array}{l}\text { Prefeitura de } \\
\text { Ceará-Mirim }\end{array}$ \\
\hline FLONA de Açu & 215 & Portaria Federal 245 de 18.07.2001 & ICMBio \\
\hline FLONA de Nísia Floresta & 174 & Decreto Federal S/N de 27.09.2001 & ICMBio \\
\hline APA de Genipabu & 1.881 & Decreto Estadual n. ${ }^{\circ} 12.620$ de 17.05 .95 & IDEMA \\
\hline APA Bonfim/Guaraíra & 42.000 & Decreto Estadual no 14.369 de 22.03 .99 & IDEMA \\
\hline APA Recifes de Corais & 180.000 & Decreto Estadual n ${ }^{\circ} 15.476$ de 06.06 .01 & IDEMA \\
\hline APA Piquiri-Uma & $40.707,45$ & Decreto Estadual n. ${ }^{\circ} 10.683$ de 06.06 .90 & IDEMA \\
\hline RDS Ponta do Tubarão & $12.946,03$ & Lei Estadual 8.349 de 18.07.2003 & IDEMA \\
\hline REFAU Tibau do Sul & 535,60 & $\begin{array}{l}\text { Decreto Municipal n }{ }^{\circ} 014 \text { de } 17.02 . \\
2006\end{array}$ & $\begin{array}{l}\text { Prefeitura de Tibau do } \\
\text { Sul }\end{array}$ \\
\hline RPPN Natural Ser Nativo & 154,29 & $\begin{array}{c}\text { Portaria Federal } \mathrm{n}^{\circ} 1.922 \text { de } 05.06 .96 \\
\text { Portaria } \mathrm{n}^{\mathrm{o}} 109 \text { de } 03.12 .1996\end{array}$ & $\begin{array}{c}\text { Cecília Gonçalves de } \\
\text { Medeiros }\end{array}$ \\
\hline RPPN Fazenda Salobro & 755,95 & Portaria Federal no 52 de 20.05 .94 & Lydia Brasileira de Brito \\
\hline RPPN Mata Estrela & $2.039,93$ & Portaria Federal n ${ }^{\circ} 20$ de 30.03 .2000 & $\begin{array}{c}\text { Destilaria Baía } \\
\text { Formosa S/A }\end{array}$ \\
\hline RPPN Santa Helena & 21,63 & Portaria Federal n ${ }^{\circ} 17$ de 08.03 .2006 & $\begin{array}{c}\text { Camarave } \\
\text { Empreendimento LTDA. }\end{array}$ \\
\hline RPPN Esperança & 500,00 & Portaria Federal no 74 de 31/08/2010 & $\begin{array}{l}\text { Queiroz Galvão Alimen- } \\
\text { tos S/A }\end{array}$ \\
\hline Parque Ecológico do Cabugy & 2.164 & $\begin{array}{c}\text { Lei Estadual } \mathrm{n}^{\circ} 5.823 \text { de } 0712.88 \text {, } \\
\text { Portaria Estadual } 446 \text { de } 31.08 .89 \\
\text { e Decreto Estadual } \mathrm{n}^{\circ} 14.813 \mathrm{de} \\
16.03 .2000\end{array}$ & IDEMA \\
\hline
\end{tabular}

FONTE: IDEMA, 2012. 
TABELA 2 - ELEMENTOS, MÓDULOS E NÚMERO DE QUESTÕES RELATIVAS A CADA MÓDULO DO QUESTIONÁRIO DE AVALIAÇÃO RÁPIDA E PRIORIZAÇÃO DA GESTÃO DE UNIDADES DE CONSERVAÇÃO-RAPID ASSESSMENT AND PRIORITIZATION OF PROTECTED AREA MANAGEMENT (RAPPAM) APLICADO NAS UNIDADES DE CONSERVAÇÃO (UCS) DO RIO GRANDE DO NORTE.

\begin{tabular}{|c|c|c|c|}
\hline $\begin{array}{c}\text { Elementos do } \\
\text { RAPPAM }\end{array}$ & $\begin{array}{l}\text { Módulos do } \\
\text { RAPPAM }\end{array}$ & $\begin{array}{l}\text { Número de } \\
\text { Questões }\end{array}$ & Pontuação Máxima \\
\hline \multirow{5}{*}{ Contexto } & 1. Perfil & 19 & \\
\hline & 2. Pressões e Ameaças & Variável & Variável \\
\hline & 3. Importância Biológica & 10 & 30 \\
\hline & 4. Importância Socioeconômica & 10 & 30 \\
\hline & 5. Vulnerabilidade & 9 & 27 \\
\hline \multirow{3}{*}{ Planejamento } & 6. Objetivos & 5 & 15 \\
\hline & 7. Amparo legal & 5 & 15 \\
\hline & 8. Desenho e planejamento da área & 6 & 18 \\
\hline \multirow{4}{*}{ Insumos } & 9. Recursos humanos & 5 & 15 \\
\hline & 10. Comunicação e informação & 6 & 15 \\
\hline & 11. Infraestrutura & 5 & 15 \\
\hline & 12. Recursos financeiros & 6 & 18 \\
\hline \multirow{3}{*}{ Processos } & 13. Planejamento & 5 & 15 \\
\hline & 14. Processo de tomada de decisão & 6 & 18 \\
\hline & 15. Pesquisa, avaliação e monitoramento & 6 & 18 \\
\hline Resultados & 16. Resultados & 12 & 36 \\
\hline \multirow{3}{*}{$\begin{array}{c}\text { Gestão Integrada } \\
\text { das UCs }\end{array}$} & 17. Desenho do sistema de UC & 14 & 42 \\
\hline & 18. Políticas de Unidades de conservação & 14 & 42 \\
\hline & 19. Contexto político & 10 & 30 \\
\hline
\end{tabular}

FONTE: WWF-Brasil (2009).

O questionário RAPPAM foi disponibilizado para o chefe de cada Unidade de Conservação, via internet e impresso. Em anexo estava incluso o pré-projeto especificando a justificativa de realização deste trabalho, seus objetivos e a metodologia aplicada, além das instruções para responder o questionário.

O questionário RAPPAM era composto por 19 módulos que exploravam os seguintes tópicos: Perfil, Pressões e Ameaças, Contexto, Efetividade de Gestão e Sistema Integrado de Unidades de Conservação. O elemento Contexto foi avaliado por meio dos módulos
3, 4 e 5; já a Efetividade de Gestão foi avaliada por meio dos elementos: planejamento (6 a 8), insumos ( 9 a 12), processos (13 a 15) e resultados da gestão (16), e a avaliação do Sistema integrado de Unidades de Conservação correspondeu aos módulos 17 a 19 (Tabela 2).

Cada módulo era composto por um conjunto de questões fechadas. Para cada alternativa de resposta dos módulos 3 a 19 foi atribuída uma pontuação: "sim" corresponde a 3 pontos, "predominantemente sim" a 2 pontos, "predominantemente não" a 1 ponto e "não" a 0 ponto. Para as questões que não foram respondidas 
por não condizerem com a realidade local da UC, foi atribuído o valor de 0 ponto. E aquelas questões que não foram respondidas devido à falta de conhecimento e interpretação da questão não foram pontuadas.

O módulo 2 do questionário RAPPAM, referente às atividades que exercem pressão e/ou ameaça às Unidades de Conservação, foi avaliado de forma qualitativa. Uma atividade que exerce pressão é aquela que tem causado impacto negativo há mais de cinco anos na Unidade de Conservação, enquanto que ameaças são atividades impactantes analisadas sob a perspectiva de sua continuidade durante os próximos cinco anos. Neste módulo, foram analisados os seguintes critérios: Tendência, Abrangência, Impacto e Permanência.

Para os demais elementos do questionário (Contexto, Planejamento, Insumos, Processos, Resultados e Gestão Integrada de UCs), foi realizado o cálculo percentual do valor obtido em relação ao valor máximo possível correspondente a cada módulo de pergunta. Foram considerados resultados altos aqueles que obtiveram valores acima de $60 \%$, médios entre $40 \%$ e $60 \%$ e baixos os resultados inferiores a $40 \%$, como estabelecido pelos estudos realizados pela WWF-Brasil.

Nesta análise descritiva, foram calculados a média e o desvio padrão de cada elemento e módulo de pergunta para melhor interpretar a tendência central e o espalhamento dos dados em torno deste ponto central.

\section{Resultados}

Das vinte e duas Unidades de Conservação que há no Estado, o questionário RAPPAM foi aplicado em oito. Destas, duas estão sob gestão federal, a Estação Ecológica do Seridó (ESEC Seridó) e a Floresta Nacional de Açu (FLONA de Açu), e seis sob gestão estadual: a Área de Proteção Ambiental de Genipabu (APA de Genipabu), Área de Proteção Ambiental de Piquiri-Una (APA de Piquiri-Una), a Área de Proteção Ambiental dos Recifes de Corais (APA dos Recifes de Corais), a Área de Proteção Ambiental Bonfim-Guaraíra (APA Bonfim-Guaraíra), o Parque Estadual Mata de Pipa (PE Mata de Pipa) e a Reserva de Desenvolvimento Sustentável Ponta do Tubarão (RDS Ponta do Tubarão).

Não foi possível aplicar o RAPPAM no Parque Ecológico Pico do Cabugy, no Parque Estadual Florêncio
Luciano e no Parque Natural Municipal Boca da Mata, pois estas UCs não têm gestores efetivos no momento. O Parque Estadual Dunas de Natal e o Parque Municipal Dom Nivaldo Monte também não foram contemplados nesse trabalho por estarem inseridos na área urbana e divergirem do padrão das perguntas exploradas pelo questionário RAPPAM. A metodologia também não foi aplicada nas cinco RPPN's, pois o questionário não é direcionado para UCs privadas. Este trabalho também não abrangeu a Floresta Nacional de Nísia Floresta, a Reserva Biológica Atol das Rocas e a Reserva Faunística de Tibau do Sul devido à dificuldade de contatar os gestores responsáveis pelas Unidades de Conservação. Por fim, o Parque Nacional da Furna Feia não foi contemplado neste trabalho por ser uma Unidade de Conservação recém-criada e não possuir ainda um gestor responsável pela área.

A presença de espécies exóticas invasoras, como Algaroba, Leucena e Caprinos, assim como a caça, são as principais atividades impactantes que têm comprometido a efetividade de gestão da ESEC Seridó. Ambas são consideradas um impacto moderado por ocorrerem nos últimos 5 a 20 anos. No entanto, a presença de espécies exóticas é uma atividade concentrada que tem aumentado ligeiramente, enquanto que a caça é uma atividade espalhada e considerada uma ameaça à UC.

Na FLONA de Açu, a principal atividade que exerce pressão sobre a UC é a expansão urbana. Este é um fenômeno localizado que tem aumentado drasticamente há mais de 100 anos, sendo considerado um impacto alto. Mas é o impacto pontual de deposição de lixo no entorno da UC que é considerado uma ameaça, apesar de moderado e de baixa probabilidade de se concretizar nos próximos cinco anos.

A especulação imobiliária e o tráfico de animais são as principais atividades que têm causado alto impacto negativo na APA de Jenipabu nos últimos cinco anos. A especulação imobiliária é uma atividade espalhada que aumentou drasticamente nos últimos 20 a 100 anos; já o tráfico de animais é uma atividade generalizada que vem ocorrendo constantemente a longo prazo.

$\mathrm{Na}$ APA Bonfim-Guaraíra, a carcinicultura e a plantação de cana-de-açúcar vêm sendo as principais atividades de alto impacto negativo. A carcinicultura é uma atividade que vem ocorrendo a médio prazo. Já a 
plantação de cana-de-açúcar tem apresentado grande abrangência e aumentado ligeiramente ao longo de 5 a 20 anos. Além do mais, ambas as atividades são consideradas ameaças à APA. A plantação de cana-de-açúcar também é uma atividade de alto impacto na APA de Piquiri-Una, sendo que a mesma vem sendo uma atividade de abrangência generalizada e permanente $(>100$ anos) que tem aumentado ligeiramente.

A pesca predatória é uma atividade de alto impacto que tem comprometido a efetividade de gestão da APA Recifes de Corais, uma vez que tem sido constante ao longo de 20 a 100 anos e abrangendo toda a área da UC marinha. No entanto, a atividade turística ainda é considerada a principal ameaça à área.

O PE Mata de Pipa sofre pressão decorrente da expansão urbana e do desmatamento. A expansão urbana, apesar de estar concentrada em uma área específica, tem aumento drasticamente há mais de 100 anos, sendo considerada uma atividade de alto impacto, ao passo que o desmatamento é uma pressão localizada que tem aumentado ligeiramente nos últimos cinco anos, sendo considerado um impacto moderado.

Por fim, a integridade da RDS Ponta do Tubarão tem sido comprometida pela construção de Parques Eólicos e pela extração de petróleo. Ambas as atividades têm aumentado drasticamente a longo prazo (20 a 100 anos) e têm apresentado uma abrangência generalizada.

As Unidades de Conservação avaliadas apresentaram média importância biológica $(59 \%)$ e socioeconômica (50\%) (Figura 2), sendo que as UCs de Uso Sustentável apresentaram valores maiores $(61 \%$ e $55 \%$, respectivamente) do que as de Proteção Integral (53\% e $35 \%)$. E as UCs federais tiveram médias menores $(33 \% \mathrm{e}$ $27 \%$, respectivamente) do que as estaduais (66\% e 58\%). Os altos níveis de Biodiversidade, a contribuição para a representatividade do sistema de UCs e a contemplação

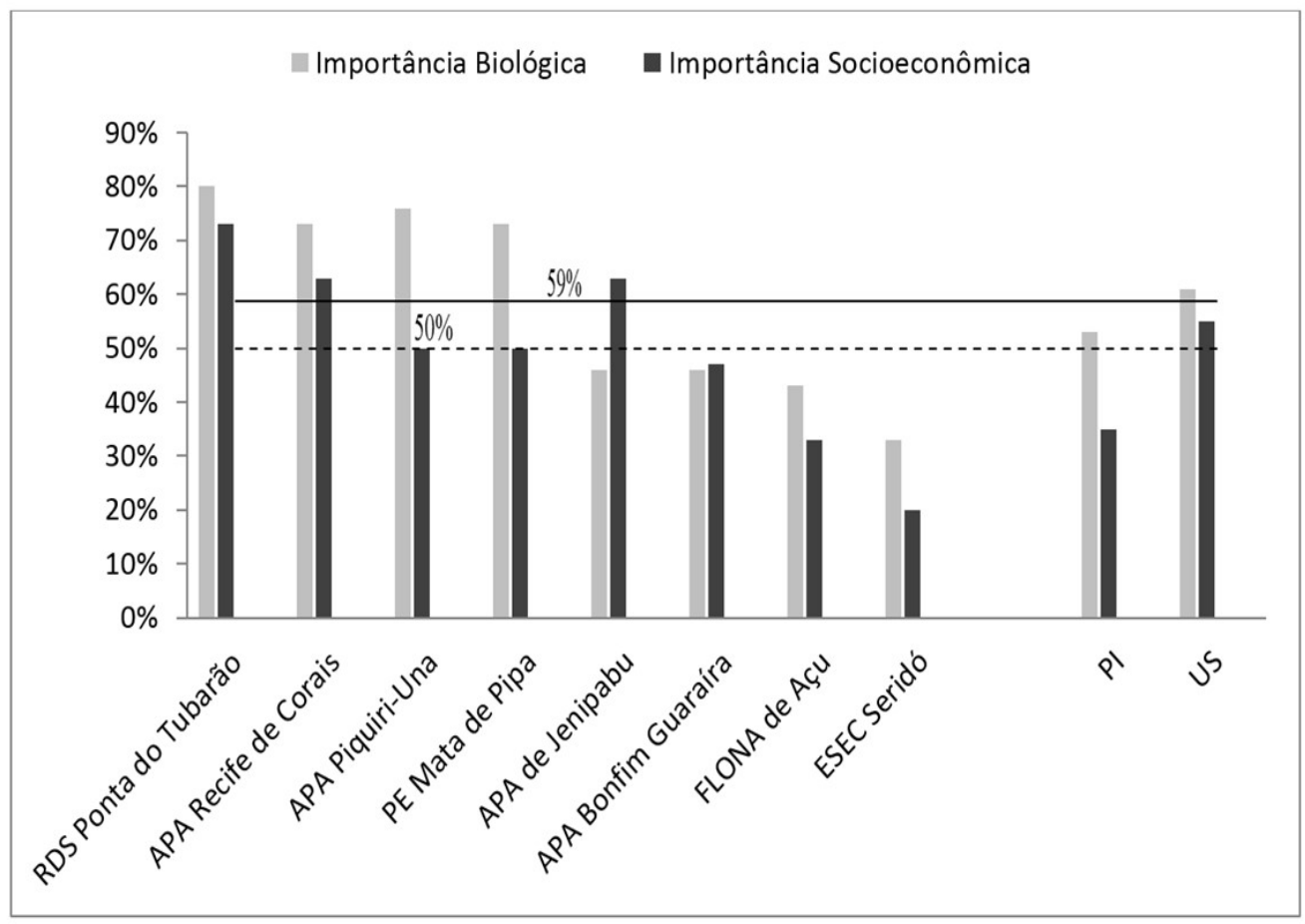

FIGURA 2 - Valores percentuais de Importância Biológica e Socioeconômica das Unidades de Conservação avaliadas por grupos de Proteção Integral (PI) e Uso Sustentável (US). Estação Ecológica (ESEC), Parque Estadual (PE), Floresta Nacional (FLONA), Área de Proteção Ambiental (APA), Reserva de Desenvolvimento Sustentável (RDS). Na linha preenchida: Média da Importância Biológica e linha pontilhada: Média da Importância Socioeconômica. 
dos ecossistemas reduzidos chaves foram os fatores que mais contribuíram para a importância biológica. Já a relevância socioeconômica dessas Unidades é decorrente do alto valor educacional/científico e recreativo da área, dos serviços ambientais prestados pelos ecossistemas preservados e pelas espécies de animais e vegetais de interesse social, cultural e econômico. Os aspectos menos relevantes foram a importância religiosa da UC e a possiblidade de desenvolvimento econômico da comunidade local a partir do uso sustentável dos recursos naturais.

As UCs avaliadas neste trabalho apresentaram vulnerabilidade mediana (54\%), em que as UCs de Proteção Integral se apresentaram pouco mais vulneráveis (55\%) do que as de Uso Sustentável (48\%); no entanto, as UCs estaduais $(60 \%)$ se apresentaram bem mais vulneráveis do que as UCs sobre gestão federal (35\%). O fácil acesso e o difícil monitoramento das Unidades de Conservação, a dificuldade de contratar funcionários, assim como o alto valor dos recursos naturais e a sua grande demanda no mercado são os principais fatores responsáveis pela vulnerabilidade dessas áreas protegidas.

Dos elementos analisados para avaliar a efetividade de gestão, o Planejamento foi o que apresentou maior relevância (63\%), seguido de Resultados (49\%), Processos (46\%) e Insumos (21\%) (Tabelas 3 e 4).

TABELA 3 - MÉDIA E DESVIO PADRÃO DOS MÓDULOS DA EFETIVIDADE DE GESTÃO POR UNIDADE DE CONSERVAÇ̃̃O FEDERAL (UCF), UNIDADE DE CONSERVAÇÃO ESTADUAL (UCE), UNIDADE DE CONSERVAÇÃO DE PROTEÇÃO INTEGRAL (UCPI) E UNIDADE DE CONSERVAÇÃO DE USO SUSTENTÁVEL (UCUS).

\begin{tabular}{|c|l|c|c|c|c|c|}
\hline Elemento & \multicolumn{1}{|c|}{ Módulo } & Média & UCF & UCE & UCPI & UCUS \\
\hline & 6. Objetivos & $83 \pm 15$ & $80 \pm 28$ & $83 \pm 12$ & $90 \pm 14$ & $80 \pm 15$ \\
\hline Planejamento & 7. Amparo Legal & $51 \pm 19$ & $73 \pm 9$ & $43 \pm 15$ & $67 \pm 19$ & $46 \pm 18$ \\
\hline & 8. Desenho e Planejamento da área & $56 \pm 11$ & $53 \pm 20$ & $56 \pm 10$ & $67 \pm 0$ & $52 \pm 10$ \\
\hline & & & & & & \\
\hline & 9. Recursos Humanos & $11 \pm 13$ & $27 \pm 9$ & $6 \pm 9$ & $27 \pm 9$ & $6 \pm 9$ \\
\hline Insumos & 10. Comunicação e informação & $40 \pm 24$ & $40 \pm 28$ & $40 \pm 26$ & $33 \pm 38$ & $42 \pm 23$ \\
\hline & 11. Infraestrutura & $36 \pm 25$ & $53 \pm 9$ & $30 \pm 27$ & $23 \pm 33$ & $40 \pm 25$ \\
\hline & 12. Recursos Financeiros & $24 \pm 23$ & $14 \pm 20$ & $28 \pm 25$ & $25 \pm 35$ & $24 \pm 23$ \\
\hline & & & & & & \\
\hline & 13. Planejamento de gestão & $74 \pm 22$ & $47 \pm 9$ & $83 \pm 15$ & $67 \pm 38$ & $77 \pm 18$ \\
\hline & 14. Tomada de decisão & $69 \pm 13$ & $81 \pm 4$ & $65 \pm 13$ & $67 \pm 16$ & $69 \pm 14$ \\
\hline & 15. Pesquisa, avaliação e monitoramento & $49 \pm 30$ & $50 \pm 24$ & $45 \pm 34$ & $39 \pm 39$ & $49 \pm 31$ \\
\hline & & & & & & \\
\hline Processos & $49 \pm 19$ & $53 \pm 4$ & $47 \pm 22$ & $36 \pm 20$ & $53 \pm 18$ \\
\hline
\end{tabular}

TABELA 4 - MÉDIA E DESVIO PADRÃO DOS ELEMENTOS DA EFETIVIDADE DE GESTÃO POR UNIDADE DE CONSERVAÇÃO FEDERAL (UCF), UNIDADE DE CONSERVAÇÃO ESTADUAL (UCE), UNIDADE DE CONSERVAÇÃO DE PROTEÇÃO INTEGRAL (UCPI) E UNIDADE DE CONSERVAÇÃO DE USO SUSTENTÁVEL (UCUS).

\begin{tabular}{cccccc}
\hline Elemento & UCF & UCE & UCPI & UCUS & Média \\
Planejamento & $68 \pm 19$ & $61 \pm 9$ & $74 \pm 10$ & $59 \pm 8$ & $63 \pm 11$ \\
Insumos & $29 \pm 2$ & $18 \pm 14$ & $16 \pm 20$ & $22 \pm 12$ & $21 \pm 13$ \\
Processos & $42 \pm 4$ & $47 \pm 5$ & $43 \pm 6$ & $47 \pm 5$ & $46 \pm 5$ \\
Resultados & $53 \pm 4$ & $47 \pm 22$ & $36 \pm 20$ & $53 \pm 18$ & $49 \pm 19$ \\
Efetividade & $51 \pm 1$ & $48 \pm 19$ & $45 \pm 9$ & $50 \pm 8$ & $49 \pm 8$ \\
\hline
\end{tabular}


A inclusão da proteção e conservação da biodiversidade nos objetivos da UC; a coerência das políticas e dos planos de ação com os objetivos da UC; o apoio e o entendimento dos objetivos da UC pelos funcionários e comunidades locais; a presença de amparo legal; a coerência de localização das UCs com os seus objetivos de conservação da biodiversidade foram os parâmetros analisados pelo elemento Planejamento que mais se destacaram. Contudo, a má utilização do uso da terra do entorno da UC, assim como a baixa conectividade entre as UCs, são as principais falhas de planejamento dessas áreas protegidas.

A facilidade de comunicação entre a Unidade de Conservação, as gerências, as diretorias e outras unidades, assim como a presença de infraestrutura para os visitantes e transporte adequado avaliados no módulo Infraestrutura, foram os indicadores que mais contribuíram na avaliação do elemento Insumos. Entretanto, o destaque é para a falta de recursos humanos suficientes para manter o manejo efetivo da Unidade de Conservação, considerando que quase todas as UCs avaliadas só tinham o gestor como funcionário atuante. As falhas nas práticas de administração financeira da Unidade, as baixas perspectivas financeiras a longo prazo e as divergências de alocação de recursos com as prioridades da UC foram outros indicadores de má gestão na avaliação dos Insumos. Todavia, as respostas presentes neste módulo foram influenciadas pela falta de informações exatas dos gestores acerca do montante de recursos disponibilizados para gestão das UCs, uma vez que eles não têm acesso a essas informações.

A presença de um inventário abrangente de recursos naturais e culturais, de um planejamento operacional que identifica as metas específicas para alcançar os objetivos de manejo, a existência de um Conselho implantado e efetivo, onde as comunidades locais participam das decisões pelas quais são afetadas, de um bom fluxo de comunicação entre os funcionários e o gestor, assim como a transparência na tomada de decisões, são elementos que proporcionam um melhor funcionamento do atual processo de gestão das UCs. Entretanto, a ausência de pesquisa acerca das questões socioeconômicas e ambientais da UC é fator crítico que compromete a efetividade de gestão.
Quanto à avaliação dos Resultados dos últimos dois anos de gestão, a prevenção e detecção de ameaças as UCs e consequente aplicação da lei, assim como o planejamento de manejo, foram as principais ações que possibilitaram a obtenção de $49 \%$ para esse módulo, enquanto que a falta de manejo da vida silvestre e dos recursos naturais, assim como a falta de projetos de recuperação e mitigação de áreas degradadas e a ausência de atividades de capacitação e supervisão dos funcionários, foram as principais falhas relatadas.

No geral, as Unidades de Conservação avaliadas obtiveram uma nota mediana de $49 \%$ para Efetividade de gestão. Este resultado foi similar para as Unidades de Conservação de gestão federal (51\%) e estadual (48\%), assim como para as UCs de Proteção Integral (45\%) e as de Uso Sustentável (50\%) (Tabela 4). Das oito Unidades de Conservação avaliadas, uma apresentou efetividade de gestão alta, a APA de Jenipabu (61\%), seis apresentaram média efetividade de gestão (entre $40 \%$ e $60 \%$ ) e uma apresentou efetividade de manejo baixa, o PE Mata de Pipa (39\%).

Quanto à avaliação do módulo de gestão integrada das UCs, os resultados revelam que o Sistema Estadual parece ser mais efetivo (57\%) do que o federal (18\%), assim como o Sistema de Unidades de Conservação é mais representativo para as Unidades de Conservação de Uso Sustentável (52\%) do que para as de Proteção Integral (32\%), sendo que duas UCs de Proteção Integral avaliadas obtiveram resultados divergentes (53\% para o PE Mata de Pipa e 11\% para a ESEC Seridó) (Tabela 5).

$\mathrm{Na}$ avaliação do módulo de gestão integrada das UCs do Estado, a presença de categorias pertinentes no Sistema de Unidade de Conservação, a representatividade da diversidade de ecossistemas da região, a presença de uma boa estrutura organizacional e de uma legislação que comtempla os objetivos das UCs foram os parâmetros mais positivos na avaliação. No entanto, a falha na proteção de áreas de alta biodiversidade e endemismo, a carência de um programa de capacitação de funcionários, a ausência de manejo rotineiro das UCs, a falta de comprometimento com a proteção de uma rede de UCs representativa e a carência de recursos financeiros suficientes para gestão efetiva do sistema de UCs foram os indicadores de maior criticidade de gestão integrada. 
TABELA 5 - MÉDIA E DESVIO PADRÃO DOS MÓDULOS DE GESTÃO INTEGRADA DAS UCS POR UNIDADE DE CONSERVAÇÃO FEDERAL (UCF), UNIDADE DE CONSERVAÇÃO ESTADUAL (UCE), UNIDADE DE CONSERVAÇÃO DE PROTEÇÃO INTEGRAL (UCPI) E UNIDADE DE CONSERVAÇÃO DE USO SUSTENTÁVEL (UCUS).

\begin{tabular}{|l|c|c|c|c|c|}
\hline Módulo & UCF & UCE & UCPI & UCUS & Média \\
\hline 17. Desenho do Sistema de UC & $24 \pm 10$ & $53 \pm 15$ & $32 \pm 22$ & $50 \pm 17$ & $46 \pm 19$ \\
\hline 18. Políticas de UC & $13 \pm 8^{*}$ & $55 \pm 14$ & $26 \pm 27$ & $51 \pm 20$ & $45 \pm 23$ \\
\hline 19. Contexto Político & $13 \pm 8^{*}$ & $64 \pm 18$ & $40 \pm 42$ & $56 \pm 25$ & $55 \pm 28$ \\
\hline Gestão Integrada das UCs & $18 \pm 9$ & $57 \pm 13$ & $32 \pm 30$ & $52 \pm 19$ & $49 \pm 6$ \\
\hline
\end{tabular}

* a média foi afetada pela falta de interpretação das questões pelo gestor.

\section{Discussão}

As atividades que exercem impactos sobre as Unidades de Conservação podem ser tanto de origem natural quanto antrópica. De acordo com Peccatiello (2007), essas alterações podem ser observadas na perda da integridade física, biológica e social do ambiente. Neste trabalho, as atividades que exerceram pressões e ameaças sobre as Unidades de Conservação analisadas estão atreladas principalmente ao uso e à coleta ilegal dos recursos naturais, assim como à conversão do uso e ocupação do solo do interior e da área do entorno da UC.

A coleta ilegal de recursos naturais, tráfico de animais, pesca e caça predatória, desmatamento e conflitos com a atividade turística foram as principais atividades impactantes relacionadas ao uso e à coleta ilegal de recursos naturais, relatadas tanto em Unidades de Conservação de Uso Sustentável quanto nas de Proteção Integral. No entanto, essa atividade se torna mais crítica em UCs de Proteção Integral, pois a mesma é criada com o objetivo maior de manter a integridade da área preservada. As restrições de uso dos recursos ambientais, ocasionalmente, se traduzem em conflitos com populações humanas que vivem no interior ou no entorno da UC, tornando essas áreas mais vulneráveis do que as UCs de Uso Sustentável, como foi relatado no trabalho de Barbosa e Santos (2008).

$\mathrm{O}$ aumento da expansão urbana, a especulação imobiliária, a construção de Parques Eólicos, a deposição de lixo no entorno da UC e o cultivo de cana-de-açúcar e de camarão são os principais conflitos advindos do direcionamento do uso do solo para outros fins que não sejam a conservação. Essas atividades foram relatadas somente nas Unidades de Conservação de Uso Sustentável. A prática da sustentabilidade em UC é bem mais complexa do que parece, pois não basta apenas categorizar o uso de recursos na Unidade: é necessário, acima de tudo, fazer com que ela seja cumprida. Trebbi et al. (2011) apontam que a falha maior das Unidades de Uso Sustentável está na falta de esclarecimento em lei da quantidade de recursos florestais e animais a serem explorados.

Diferentemente dos estudos realizados no Estado do Pará (WWF-Brasil, SEMA-PA \& ICMBIO, 2011), Amapá (WWF-Brasil, IEF-AP, SEMA-AP \& ICMBIO, 2009), Rondônia (WWF-Brasil, SEDAM-RO \& ICMBIO, 2011) e Acre (WWF-Brasil, SEMA-AC, SEF-AC \& ICMBIO, 2009), as Unidades de Conservação de Uso Sustentável neste trabalho apresentaram média maior de importância biológica do que as de Proteção Integral. Este resultado pode ser reflexo da falta de informações concretas ou desatualização de documentos técnicos, como o plano de manejo das UCs de Proteção Integral avaliadas (PE Mata da Pipa e ESEC Seridó). Documentos estes que dispõem acerca da presença de espécies endêmicas, ameaçadas de extinção e espécies-chave, indicadores de importância biológica. Para o IBAMA (2002), o Plano de Manejo é o principal documento técnico norteador das atividades a serem desenvolvidas na UC e, na falta ou desatualização deste documento, as UCs ficam à mercê do desconhecido. Das oito Unidades de Conservação avaliadas, apenas três continham Plano de Manejo e este pode ser um dos principais entraves para a efetividade do Sistema de Unidades de Conservação Potiguar. 
A diferença de importância socioeconômica é visível entre os grupos de manejo das UCs. Neste estudo, as Unidades de Uso Sustentável apresentaram maior importância socioeconômica do que as de Proteção Integral. Estes resultados são similares com os obtidos pelos questionários RAPPAM aplicados no Pará (WWF-Brasil, SEMA-PA \& ICMBIO, 2011), Amazonas (WWF-Brasil, SDS-AM \& ICMBIO, 2011), Acre (WWF-Brasil, SEMA-AC, SEF-AC \& ICMBIO, 2009) e Amapá (WWF-Brasil, IEF-AP, SEMA-AP \& ICMBIO, 2009). No entanto, os principais indicadores que proporcionaram estes resultados às UCs de Uso Sustentável estão atrelados ao valor educacional e científico da área, assim como dos serviços ambientais prestados pelos ecossistemas preservados. O uso e manejo sustentável dos recursos naturais nas UCs ainda é muito teórico e pouco aplicável. Teixeira (2005), em seus estudos, mostra a possibilidade de realização de desenvolvimento sustentável da agricultura local realizada na APA de Guaraqueçaba. Queiroz (2005) ressalta também a importância que o manejo sustentável da pesca de pirarucu teve na RDS Mamirauá, no Amazonas. Estes estudos demostram a possibilidade de alcançar a sustentabilidade das atividades econômicas em Unidades de Conservação e, como consequência, o alcance de uma maior efetividade na conservação dessas áreas protegidas.

As UCs avaliadas apresentaram média efetividade de gestão (49\%). Este percentual é o mesmo obtido com a aplicação do RAPPAM nas Unidades de Conservação Federais Brasileiras (WWF-Brasil \& ICMBIO, 2012), apesar de ter sido maior do que os valores alcançados pelas UCs federais e estaduais do Amazonas (41\%) (WWF-Brasil, SDS-AM \& ICMBIO, 2011), Pará (36\%) (WWF-Brasil, SEMA-PA \& ICMBIO, 2011), Rondônia (35\%) (WWF-Brasil, SEDAM-RO \& ICMBIO, 2011) e das UCs estaduais do Amapá (41\%) (WWF-Brasil; IEF-AP; SEMA-AP; ICMBIO, 2009) e Mato Grosso do Sul (44\%) (WWF-Brasil \& IMASUL, 2011). Neste estudo, o planejamento do Sistema de Unidades de Conservação foi o elemento de gestão que mais contribuiu para a sua efetividade. Este resultado também foi observado com a aplicação do RAPPAM em todos os estados em que ele foi aplicado.

A carência de disponibilidade de recursos humanos e financeiros para gerir uma Unidade de Conservação adequadamente é uma das lacunas de gestão mais comuns. De acordo com Paduá (2011), o Brasil ainda é um dos países do mundo que possui menos funcionários por hectares protegidos e menos recursos financeiros. A WWF, em parceria com o ICMBio, repetiu em 2010 um estudo realizado primeiramente em 2005 para averiguar a efetividade de gestão das Unidades de Conservação Federais do Brasil. Como resultado, obtiveram que, apesar da significativa melhora nos últimos seis anos, as limitações dos recursos financeiros e humanos ainda são o principal entrave para consolidar as Unidades de Conservação Federais (WWF-Brasil \& ICMBIO, 2012). Neste trabalho, os resultados não são diferentes, já que a categoria Insumos do questionário RAPPAM apresentou os menores resultados ao avaliar os recursos financeiros e humanos.

A facilidade de comunicação entre as diferentes Unidades de Conservação, assim como a presença de infraestrutura para visitantes e pesquisadores, são fatores que otimizam o sistema de gestão e são o diferencial de muitas UCs do país. As Unidades de Conservação estaduais, por exemplo, apresentam uma infraestrutura padrão denominada Ecoposto, que fornece apoio necessário para pesquisadores, visitantes, polícia ambiental e administração. Esta infraestrutura está disponível em quatro das seis Unidades de Conservação Estaduais avaliadas. As duas UCs sob gestão federal avaliadas também contam com infraestrutura adequada.

As práticas de gestão desenvolvidas nas Unidades de Conservação revelam que a estruturação de um conselho gestor implantado em sete das oito UCs avaliadas auxilia em uma maior participação comunitária na gestão da UC, assim como numa melhor transparência das diretrizes de gestão que têm fortificado as atividades de gerenciamento dessas áreas. No entanto, a falta de investimentos em pesquisas ecológicas desenvolvidas nas Unidades de Conservação é fator crítico da atividade de gestão, pois as pesquisas de caráter científico, de acordo com Mikich (2006, apud Ferreira \& Figueiredo, 2012), só têm a somar na gestão dessas áreas; todavia, a avaliação delas se torna subjetiva quando não existe uma valorização dessas atividades.

Neste trabalho, o Sistema Estadual de Unidades de Conservação mostrou-se mais efetivo (57\%) do que o Sistema Federal (18\%). Este resultado pode estar 
relacionado à ampliação da rede de áreas protegidas do Estado sob gestão do IDEMA, assim como dos esforços para consolidá-las, uma vez que têm sido implementados Conselhos gestores em todas as UCs. Já os avanços de gestão das UCs a cargo do ICMBio vêm ocorrendo de forma pouco visível. A diferença do Sistema de Unidades de Conservação também é perceptível entre os grupos de manejo (52\% para as de Uso Sustentável e 32\% para as de Proteção Integral). No Estado, a preferência pela criação de Unidades de Conservação de Uso Sustentável do que de Proteção Integral ocorre uma vez que essas áreas apresentam maior flexibilidade no uso dos recursos naturais e de ocupação.

\section{Conclusões}

As Unidades de Conservação do Rio Grande do Norte estão inseridas em um contexto ambiental, econômico e político de grandes desafios a serem enfrentados e oportunidades a serem exploradas, independente da esfera de gestão ou da categoria de manejo. As fragilidades do Sistema de Unidades de Conservação Potiguar se refletem principalmente na carência de investimento em recursos humanos, financeiros e de pesquisa, na falta de aplicação dos princípios da sustentabilidade na utilização dos recursos naturais como provedores do desenvolvimento econômico local e no pouco comprometimento social e político em manter um sistema de áreas protegidas conectadas. Contudo, a gestão das Unidades de Conservação encontra potencialidades no momento em que conta com um planejamento operacional que abrange tanto valores sociais quanto econômicos e ambientais, que é otimizado com a implantação de uma boa infraestrutura física da UC e na participação comunitária representada pelos conselhos gestores.

As principiais medidas para aumentar a efetividade das UCs potiguares podem ser agrupadas nos seguintes temas: a) Planejamento - Elaborar planos de manejo para as UCs que ainda não têm; b) Pesquisa - Estabelecer parcerias com instituições públicas e privadas para desenvolver pesquisas nas UCs; c) Monitoramento - Estabelecer avaliação periódica de efetividade de gestão das UCs e desenvolver programas de fiscalização; d) Recursos Humanos - Viabilizar concurso público para lotação de funcionários nas UCs, realizar oficinas de capacitação para os funcionários e acompanhar o seu desempenho; e) Recursos Financeiros - Possibilitar o acesso direto dos recursos aos gestores e captar recursos financeiros externos; f) Gestão - Planejar e implementar gestão integrada das UCs, estabelecer a zona de amortecimento da UCs e elaborar programas de uso dos recursos naturais do interior e entorno das UCs; g) Responsabilidade Social - Estimular o desenvolvimento de atividades sustentáveis nas UCs, implementar programas de educação ambiental com a comunidade do interior e do entorno das UCs e envolver a comunidade do interior/entorno da UC no planejamento e monitoramento da UC, com vínculo empregatício; h) Divulgação - Criar mecanismos de divulgação das UCs, assim como das atividades de pesquisa desenvolvidas nessas áreas protegidas.

Incorporar o processo de avaliação como parte integral da gestão de áreas protegidas no Estado é o próximo desafio. Este não é um trabalho pontual, seu princípio de diagnóstico é cíclico e seus resultados devem ser revisados periodicamente. Essas avaliações periódicas poderão auxiliar a realização de ajustes para melhorar a gestão das Unidades de Conservação já existentes.

\section{Agradecimentos}

Agradecemos ao ICMBIO e ao IDEMA por participarem ativamente dos preenchimento dos formulários. Aos revisores da revista Desenvolvimento e Meio Ambiente pelas correções e sugestões, todas foram de extrema importância para o aprimoramento desse artigo. Ao CNPq pela bolsa de produtividade para EMV 


\section{Referências}

Barbosa, R. S.; Santos, F. D. Unidades de conservação, conflitos socioambientais e o encurralamento das populações locais no Norte de Minas. In: Anais do XXVI Congresso da Sociedade Brasileira de Economia, Administração e Sociologia Rural. Rio Branco, 2008.

Cifuentes, A. M.; Izurieta, A.; Faria, H. H. Medición de la efectividad de manejo de areas protegidas. Turrialba: WWF/ IUCN/GTZ, 2000.

Ervin, J. Metodologia do WWF para avaliação rápida e a priorização do manejo de Unidades de Conservação - RAPPAM. Tradução: WWF-Brasil São Paulo: WWF-Brasil, 2003.

Farias, F. H. C.; Medeiros, R. Unidades de Conservação do Rio Grande do Norte e a meta dos $10 \%$ de áreas protegidas estabelecidas pela Convenção da Biodiversidade (2002). In: Anais do VII Congresso Brasileiro de Unidades de Conservação. Natal, out. 2012.

Ferreira, E. T.; Figueiredo, C. A. A. A gestão e a importância das pesquisas científicas nas Unidades de Conservação: o caso da área de proteção ambiental de Gericinó-Mendanha. In: Anais do VII Congresso Brasileiro de Unidades de Conservação. Natal, out. 2012.

Hocking, M.; Stolton, S.; Dudley, N. Evaluating Effectiveness: a framework for assessing the management of protected areas. Gland, Switzerland e Cambridge, UK: IUCN, 2000.

IBAMA - Instituto Brasileiro do Meio Ambiente e dos Recursos Naturais Renováveis. Roteiro metodológico de planejamento: parque nacional, reserva biológica, estação ecológica. 2002. Disponível em: <http://www.icmbio.gov.br/portal/images/ stories/imgs-unidadescoservacao/roteiroparna.pdf $>$. Acesso em: 01 set. 2012.

IBGE - Instituto Brasileiro de Geografia e Estatística. Informações do Rio Grande do Norte. 2010. Disponível em: <http:// www.ibge.gov.br/estadosat/perfil.php?sigla=rn $>$. Acesso em: 04 set. 2012.

IDEMA - Instituto de Desenvolvimento Sustentável e Meio Ambiente. Unidades Estaduais de Conservação Ambiental do $R N$. Disponível em: < http://www.idema.rn.gov.br/Conteudo. asp?TRAN=ITEM \&TARG $=334 \&$ ACT $=\&$ PAGE $=0 \&$ PARM $=\& \mathrm{LBL}=$ Unidades + de + Conserva $\% \mathrm{E} 7 \% \mathrm{E} 3 \mathrm{o}>$. Acesso em: 06 set. 2012.

Paduá, M .T. J. Do Sistema Nacional de Unidades de Conservação. In: Medeiros, R.; Araújo, F. F. S. (Orgs.). Dez anos do Sistema Nacional de Unidades de Conservação da Natureza: lições do passado, realizações presentes e perspectivas para o futuro. Brasília: MMA, 2011.

Peccatiello, A. F. O. Análise ambiental da capacidade de carga antrópica na trilha principal do Circuito Pico do Pião - Parque Estadual do Ibitipoca, MG. Juiz de Fora, Dissertação (Especialização em Análise Ambiental) - Universidade Federal de Juiz de Fora, 2007.

Prates, A. P. L. Desafios para atingir as metas e o status do Sistema Nacional de Unidades de Conservação-SNUC. Natal, 20 set. 2012. Palestra proferida no VII Congresso Brasileiro de Unidades de Conservação.

Queiroz, H. L. A reserva de desenvolvimento sustentável Mamirauá. Estudos Avançados, 19(54), 183-202, 2005.

Schaik, C. V.; Rijksen, H. D. Projetos integradores de conservação e desenvolvimento: problemas e potenciais. In: Terbogh, J.; Schaik, C. V.; Davenport, L.; Rao, M. (Orgs.). Tornando os parques eficientes: estratégias para conservação da natureza nos trópicos. Curitiba: UFPR, 2002. p. 37-51.

Teixeira, C. O desenvolvimento sustentável em Unidades de Conservação: a "naturalização" do social. Revista Brasileira de Ciências Sociais, 20(59), 51-66, 2005.

TNC - The Nature Conservency. Medición de Logros: Manual de la Tabla de Puntación ("Scorecard") para la Consolidación de Sitios. Arlington, VA, USA: The Nature Conservancy, 2007.

Trebbi, L. S.; Vianna, B. V. G.; Piana, B. M.; Freitas, D. M.; Souza, R. A. Desmatamento em áreas protegidas da Caatinga. In: Anais do V Simpósio Regional de Geoprocessamento e Sensoriamento Remoto. Feira de Santana, 2011.

WWF-Brasil; Instituto Florestal. Implantação do RAPPAM em Unidades de Conservação do Instituto Florestal e da Fundação Florestal de São Paulo. 2004. Disponível em: <http:// www.mma.gov.br/estruturas/pda/_arquivos/prj_mc_061_pub_ liv_003_uc.pdf>. Acesso em: 13 ago. 2012.

; IBAMA. Efetividade de gestão das Unidades de Conservação Federais do BRASIL: implementação do Método Rappam - Avaliação Rápida e Priorização da Gestão de Unidades de Conservação. 2007. Disponível em: <http://www. icmbio.gov.br/portal/images/stories/documentos/2\%20-.pdf $>$. Acesso em: 13 ago.2012.

; ICMBIO. Efetividade de gestão das Unidades de Conservação Federais do Brasil: resultados de 2010. 2012. Disponível em: <http://observatorio.wwf.org.br/site_media/ 
upload/gestao/documentos/Rappam_federal_2010.pdf>. Acesso em: 13 ago. 2012.

; IEF-AP; SEMA-AP; ICMBIO. Efetividade de gestão das Unidades de Conservação no Estado do Amapá. 2009. Disponível em: <http://d3nehc6yl9qzo4.cloudfront.net/downloads/rappam_amapa_1.pdf $>$. Acesso em: 13 ago. 2012.

; SEMA-AC; SEF-AC. Efetividade de gestão das Unidades de Conservação no Estado do Acre. 2009. Disponível em: <http://d3nehc6yl9qzo4.cloudfront.net/downloads/ rappam_acre.pdf $>$. Acesso em: 13 ago. 2012.

; SEMA-MT; ICMBIO. Efetividade de gestão das Unidades de Conservação no Estado do Mato Grosso. 2009. Disponível em: <http://d3nehc6y19qzo4.cloudfront.net/downloads/rappam_matogrosso.pdf $>$. Acesso em: 13 ago. 2012.

; SEMAC-MS; IMASUL-MS; ICMBIO. Efetividade

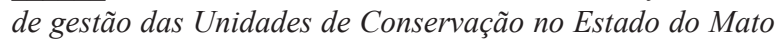

Grosso do Sul. 2011. Disponível em: <http://d3nehc6yl9qzo4. cloudfront.net/downloads/rappam_ms0912_web.pdf $>$. Acesso em: 13 ago. 2012.

; SDS-AM; ICMBIO. Efetividade de gestão das Unidades de Conservação do Estado do Amazonas. 2011. Disponível em: <http://d3nehc6yl9qzo4.cloudfront.net/downloads/ rappam_am_260811_baixa.pdf>. Acesso em: 13 ago. 2012.

; SEDAM-RO; ICMBIO. Efetividade de gestão das Unidades de Conservação do Estado de Rondônia. 2011. Disponível em: $<$ http://d3nehc6yl9qzo4.cloudfront.net/downloads/rappam_ro_280211_baixa.pdf $>$. Acesso em: 13 ago. 2012.

; SEMA-PA; ICMBIO. Efetividade de gestão das Unidades de Conservação do Estado do Pará. 2011. Disponível em: <http://d3nehc6yl9qzo4.cloudfront.net/downloads/ rappam_pa15072011_baixa.pdf>. Acesso em: 13 ago. 2012. 